'Instituto de Sociología, Pontificia Universidad Católica de Chile. aSocióloga, Doctora en Sociología.

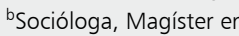
Sociología.

'Cientista Política, Magíster en Sociología.

Este artículo es producto de un estudio financiado por la Comisión Nacional de Investigación Científica y Tecnológica del Gobierno de Chile-CONICYT, a través del proyecto FONDECYT 1120331

"Eventos estresantes que ocurren al envejecer: cómo las relaciones familiares y los recursos sociales moderan e impacto en el bienestar de los mayores". También se contó con financimiento del Concurso de Investigación Adulto Mayor y Envejecimiento VRI Pontificia Universidad Católica de Chile.

Recibido el 3 de abril de 2014, aceptado el 27 de agosto de

Correspondencia a:

M. Soledad Herrera Instituto de Sociología, Pontificia Universidad Católica de Chile. Av. Vicuña Mackenna 4860, Macul, Santiago de Chile. Teléfono: (56) 23544651 mherrepo@uc.cl.

\section{Validación de un test breve para el diagnóstico de capacidad funcional en adultos mayores en Chile}

\author{
M. SOLEDAD HERRERA P. ${ }^{1, a}$, PAULA SALDÍAS $^{\mathrm{b}}$, NATALIA TESTA $^{\mathrm{c}}$
}

\section{Validation of a brief screening test to assess functional capacity in Chilean older people}

Background: The Pfeffer Functional Activities Questionnaire (PFAQ) is a validated instrument used in Chile to diagnose dependency in older people. Aim: To suggest a shorter and easier version of the PFAQ, with appropriate levels of sensitivity and specificity, which may be applied to the general older population. Material and Methods: Three Chilean surveys applied to older people were used, namely the Survey of Life Events of the Elderly, $(n=1,371)$ and the 2010 and 2013 National Surveys of Quality of Life of Older People $(n=1,954$ and 2,644 respectively). To evaluate the instrument, we performed correlation analysis between items and principal components analysis. We calculated internal consistency using Cronbach alpha, we computed sensitivity and specificity indicators, and analyzed convergent and predictive validity. Results: After constructing different scales, the one that gave more information and had the best balance between sensitivity and specificity was chosen. Items with less weight factor and greater gender bias were eliminated, keeping seven questions from the original PFAQ. Reliability was verified using Cronbach alpha's coefficient and from correlations between the new instrument and key variables. Conclusions: According to our goals, the proposed scale is solely conformed by items that do not have gender bias. It discriminates in the general population adequately, allowing the design of a shorter test, of easier implementation.

(Rev Med Chile 2014; 142: 1128-1135)

Key words: Activities of Daily Living; Dependency (Psychology); Elderly.
$\mathrm{D}$ ebido a la disminución en la tasa de fecundidad y el aumento en la esperanza de vida, la proporción de adultos mayores está aumentando rápidamente en casi todos los países ${ }^{1}$. Chile está envejeciendo a ritmo acelerado, donde $15 \%$ tiene 60 o más años, siendo dentro de América Latina el tercer país más envejecido, después de Argentina y Uruguay ${ }^{2}$. En 2011, la esperanza de vida al nacer en Chile era de 79,3 años, sólo 2 años menos que el promedio de la $\mathrm{OCDE}^{3}$.

Junto con el aumento de la esperanza de vida aparece un deterioro físico progresivo asociado a enfermedades crónicas y/o degenerativas que produce la pérdida de autonomía del adulto ma- yor, declinación de sus capacidades funcionales y la necesidad de cuidados y resguardo por parte de su entorno, lo que se traduce en un aumento de la dependencia. El concepto de dependencia alude a la incapacidad para realizar una serie de actividades de la vida diaria, que implica la necesidad de la ayuda de otras personas ${ }^{4}$. Para la medición de la dependencia funcional se utilizan principalmente dos tipos de escalas que miden la capacidad para realizar actividades básicas e instrumentales de la vida diaria",5. Las escalas de "actividades básicas de la vida diaria" más conocidas son el "Índice de Katz"6 y el "Índice de Barthel"7, las cuales miden capacidad física. Entre las escalas de "Actividades 
Instrumentales de la vida diaria"cabe mencionar el "Índice de Lawton"8 y el "Cuestionario de Actividades funcionales de Pfeffer (en adelante PFAQ $)^{9,10}$, que incluyen actividades de mayor complejidad que las actividades básicas de la vida diaria. Las primeras son más sensibles para detectar alta dependencia y usualmente se usan en contextos más medicalizados (adultos mayores institucionalizados, hospitalizados o con enfermedades invalidantes), mientras que las segundas pueden ser usadas en población general.

En la presente investigación se pretende evaluar las propiedades psicométricas del PFAQ en Chile, y proponer una versión más reducida de la escala. PFAQ nunca ha sido validado en Chile como un instrumento para detectar disminución de capacidad funcional aplicado en encuestas generales en personas mayores. Los objetivos de esta validación son: i) evaluar sesgos de género que puedan tener algunas preguntas; ii) evaluar la capacidad de discriminación de los ítems, en particular, detectar ítems con muy poca variabilidad; iii) evaluar la unidimensionalidad de la escala (validez de constructo) y su consistencia interna; iv) acortar el cuestionario que tiene originalmente 11 ítems, pues se quiere proponer una escala que pueda aplicarse en encuestas más largas, donde se tratan también otros temas; v) comparar la escala reducida con la original, evaluando sus propiedades métricas.

\section{Material y Métodos}

\section{Fuente de datos}

Se utilizan tres encuestas chilenas aplicadas presencialmente a población adulta mayor residente en sus viviendas particulares y que pueden contestar las preguntas a un encuestador. La primera es la "Encuesta sobre Eventos Vitales de los Adultos Mayores" del Proyecto Fondecyt 1120331, muestra aleatoria probabilística de 1.371 adultos de 60 o más años en el Gran Santiago el año 2012(EEV2012). La segunda y tercera es la "Encuesta Nacional de Calidad de Vida en la Vejez" de los años $2010(\mathrm{n}=1.954)$ y $2013(\mathrm{n}=2.644)$, respectivamente (ECV2010 y ECV2013). Tienen muestra aleatoria probabilística multietápica similar a EEV2012, ampliándose a cobertura nacional, siendo representativas de $86 \%$ de la población que vive en pueblos o ciudades de más de 10.000 habitantes en Chile. Estas encuestas han contado con la aprobación del Comité de Ética del Instituto de Sociología de la Pontificia Universidad Católica de Chile.

Las características sociodemográficas de las tres encuestas son similares; en el total de las tres encuestas $65 \%$ es mujer; $61 \%$ tiene entre $60 \mathrm{y}$ 74 años; 59\% tiene educación básica (8 años de estudios o menos), $28 \%$ educación secundaria y $13 \%$ algo de educación superior; $16 \%$ declara tener mala salud, $42 \%$ regular salud y $41 \%$ buena salud.

\section{Instrumentos}

El Cuestionario PFAQ es una herramienta de screening para evaluar la independencia en el desempeño de actividades de la vida diaria, diseñado para estudios de comunidades de personas con envejecimiento normal y personas que presentan alteraciones funcionales leves. El test puede ser respondido por el entrevistado o un informante confiable ${ }^{4}$. La versión original del año $1982^{9}$ tenía 10 ítems sobre actividades ante las cuales se preguntaba acerca de la habilidad para desarrollarlas en una escala de 6 respuestas que variaban según de qué actividad se tratase: actividades financieras, hacer trámites, compras, practicar algún hobbie, calentar agua para té/café, preparar la comida, mantenerse al tanto de los acontecimientos, comprender un programa de televisión o lectura, recordar acontecimientos, movilizarse fuera del barrio.

En Chile, Quiroga, Albala y Klaasen ${ }^{11}$ validaron este instrumento como un test de tamizaje para el diagnóstico de demencia en personas de 65+ años. La versión en español que utilizaron (la misma que se ocupa en el presente estudio) constó de un total de 11 ítems, para cada uno de los cuales existieron 4 respuestas posibles: $0=$ Sí es capaz o podría hacerlo; $1=$ Con alguna dificultad; $2=$ Necesita ayuda; 3 = No es capaz. El puntaje total se calcula como la suma de los puntajes de cada ítem (0 a 33 puntos). En base a un análisis de sensibilidad y especificidad y curvas ROC, y teniendo también en consideración las diferencias por educación en el desempeño cognitivo, los autores llegan a la conclusión de que el mejor punto de corte para diagnosticar deterioro cognitivo es de $>=6$.

\section{Estadística}

Para llevar a cabo la evaluación del instrumento para el caso chileno, se siguieron determinadas etapas. En primer lugar, se cruzaron todas las 
variables con género y nivel educacional, pues se quería explorar si hay ciertas preguntas que tienen sesgo cultural de género o por nivel socioeconómico; también se cruzaron con edad, pues se esperaba que todas las actividades disminuyeran en capacidad con la edad. La validez de constructo fue chequeada con correlación entre ítems y análisis de componentes principales. A partir de los resultados se construyeron distintas escalas con menor cantidad de ítems, donde se analizó la consistencia interna (correlaciones con la escala y Coeficiente Alfa de Cronbach), se calcularon estadísticos de sensibililidad y especificidad teniendo como gold standard el punto de corte en 6 puntos propuesto por Quiroga, Albala y Klaasen ${ }^{11}$; y se cruzaron con variables supuestamente relacionadas, ya sea porque se supone que miden aspectos similares (validez convergente) o porque predicen otros comportamientos (validez predictiva).

\section{Resultados}

En la Tabla 1 se tienen las frecuencias de todos los ítems de PFAQ para la muestra agregada de las encuestas analizadas. La mayoría de las respuestas están concentradas en "sí es capaz o podría hacer- lo". Las preguntas que tienen menor variabilidad son: saludar a sus amigos adecuadamente, manejar su propio dinero y calentar agua/ apagar la cocina.

Al cruzar con descriptores sociodemográficos (Tabla 2), se observa que todos los ítems se asocian con edad en la dirección esperada, disminuyendo significativamente el porcentaje de los que son capaces de realizar las actividades funcionales cuando se tiene 75 o más años. Todas las actividades se asocian con educación, donde los que tienen educación media o superior tienen menos dificultades para realizarlas, en comparación con los que tienen educación básica. Respecto de género, casi no se observan diferencias, excepto en F8 "preparar la comida", donde $91 \%$ de las mujeres señala que es capaz de realizarlo, disminuyendo a $79 \%$ en el caso de los hombres.Por esto se decidió eliminar este ítem en la escala PFAQ modificada.

Todos los análisis factoriales realizados en las tres encuestas analizadas arrojaron resultados unidimensionales (Tabla 3), pues todos los ítems de la escala están altamente correlacionados entre sí. Consistentemente, los pesos factoriales más bajos en ECV2010 y EEV2012 estuvieron en los ítems F9 "poner atención, entender, discutir un programa de radio, televisión o un artículo del periódico"

Tabla 1. Distribución de frecuencia de las preguntas PFAQ

\begin{tabular}{|c|c|c|c|c|c|c|}
\hline & Es capaz de.... & $\begin{array}{l}\text { Sí es capaz o } \\
\text { podría hacerlo }\end{array}$ & $\begin{array}{l}\text { Con alguna } \\
\text { dificultad }\end{array}$ & $\begin{array}{l}\text { Necesita } \\
\text { ayuda }\end{array}$ & $\begin{array}{l}\text { No es } \\
\text { capaz }\end{array}$ & Total \\
\hline F1 & Manejar su propio dinero & $92,1 \%$ & $3,6 \%$ & $2,8 \%$ & $1,5 \%$ & $100 \%$ \\
\hline F2 & $\begin{array}{l}\text { Calentar agua para café o té y apagar la } \\
\text { cocina }\end{array}$ & $91,4 \%$ & $3,9 \%$ & $2,3 \%$ & $2,4 \%$ & $100 \%$ \\
\hline F3 & Manejar sus propios medicamentos & $90,1 \%$ & $4,3 \%$ & $3,6 \%$ & $2,0 \%$ & $100 \%$ \\
\hline F4 & Quedarse solo en la casa sin problemas & $89,9 \%$ & $5,1 \%$ & $2,5 \%$ & $2,5 \%$ & $100 \%$ \\
\hline F5 & $\begin{array}{l}\text { Mantenerse al tanto de los acontecimientos } \\
\text { y de lo que pasa en el país }\end{array}$ & $87,7 \%$ & $7,6 \%$ & $2,3 \%$ & $2,5 \%$ & $100 \%$ \\
\hline F6 & Hacer las compras & $84,1 \%$ & $6,6 \%$ & $5,9 \%$ & $3,4 \%$ & $100 \%$ \\
\hline F7 & $\begin{array}{l}\text { Andar por el vecindario y encontrar } \\
\text { el camino de vuelta a casa }\end{array}$ & $89,2 \%$ & $4,9 \%$ & $3,3 \%$ & $2,6 \%$ & $100 \%$ \\
\hline F8 & Preparar la comida & $86,3 \%$ & $5,7 \%$ & $3,9 \%$ & $4,1 \%$ & $100 \%$ \\
\hline F9 & $\begin{array}{l}\text { Poner atención, entender, discutir un pro- } \\
\text { grama de radio, televisión o un artículo del } \\
\text { periódico }\end{array}$ & $86,9 \%$ & $8,7 \%$ & $2,8 \%$ & $1,6 \%$ & $100 \%$ \\
\hline F10 & $\begin{array}{l}\text { Recordar compromisos y acontecimientos } \\
\text { familiares }\end{array}$ & $81,0 \%$ & $12,2 \%$ & $4,7 \%$ & $2,1 \%$ & $100 \%$ \\
\hline F11 & Saludar a sus amigos adecuadamente & $92,3 \%$ & $5,0 \%$ & $1,8 \%$ & $0,9 \%$ & $100 \%$ \\
\hline
\end{tabular}

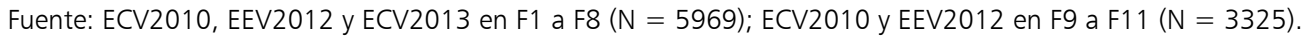


Tabla 2. Asociaciones bivariadas de los ítems PFAQ con descriptores sociodemográficos (\% "sí es capaz o podría hacerlo")

\begin{tabular}{|c|c|c|c|c|c|c|c|}
\hline & \multicolumn{2}{|c|}{ Sexo } & \multicolumn{2}{|c|}{ Edad } & \multicolumn{3}{|c|}{ Nivel de Educación } \\
\hline & Hombre & Mujer & $60-74$ & 75 y más & Básica o menos & Media & Superior \\
\hline F1 & $92,2 \%$ & $92,0 \%$ & $95,1 \%$ & $87,6 \%_{b}$ & $89,8 \%{ }_{a}$ & $95,8 \%_{b}$ & $95,0 \%_{b}$ \\
\hline F2 & $89,7 \%$ & $92,3 \%_{b}$ & $95,4 \%$ & $85,3 \%{ }_{b}$ & $89,2 \%$ & $94,9 \%$ & $94,3 \%_{b}$ \\
\hline F3 & $88,3 \%{ }_{a}$ & $91,1 \%_{b}$ & $94,8 \%{ }_{a}$ & $83,0 \%_{b}$ & $87,4 \%{ }_{a}$ & $94,4 \%{ }_{b}$ & $93,6 \%_{b}$ \\
\hline F4 & $89,7 \%$ & $90,1 \%{ }_{a}$ & $94,2 \%$ & $83,4 \%_{b}$ & $87,2 \%$ & $94,0 \%$ & $94,0 \%_{b}$ \\
\hline F5 & $87,3 \%{ }_{a}$ & $87,9 \%$ & $92,7 \%$ & $80,1 \%_{b}$ & $84,6 \%$ & $91,7 \%{ }_{b}$ & $93,6 \%_{b}$ \\
\hline F6 & $84,7 \%$ & $83,7 \%$ & $91,0 \%$ & $73,5 \%$ & $79,8 \%$ & $89,7 \% \%_{b}$ & $91,9 \%_{b}$ \\
\hline F7 & $90,3 \%$ & $88,7 \%$ & $94,7 \%$ & $80,8 \%_{b}$ & $85,7 \%$ & $94,3 \%_{b}$ & $94,6 \%_{b}$ \\
\hline F8 & $78,6 \%$ & $90,5 \%$ & $92,2 \%$ & $77,3 \%_{b}$ & $83,7 \%$ & $90,5 \%$ & $89,6 \%_{b}$ \\
\hline F9 & $88,4 \%$ & $86,0 \%_{b}$ & $91,7 \%$ & $79,3 \%{ }_{b}$ & $82,1 \%$ & $93,3 \% \%_{b}$ & $94,2 \%_{b}$ \\
\hline F10 & $81,5 \%$ & $80,7 \%$ & $87,3 \%_{a}$ & $71,1 \%_{b}$ & $75,6 \%$ & $87,6 \%_{b}$ & $90,6 \%_{b}$ \\
\hline F11 & $93,2 \%$ & $91,8 \%$ & $95,5 \%$ & $87,2 \%_{b}$ & $89,5 \%$ & $95,9 \%$ & $96,9 \%_{b}$ \\
\hline
\end{tabular}

Fuente: ECV2010, EEV2012 y ECV2013 en F1 a F8 ( $n=5969) ; ~ E C V 2010$ y EEV2012 en F9 a F11 ( $N=3325)$. Nota: Los valores de la misma fila y subtabla que no comparten el mismo subíndice son significativamente diferentes en $p<, 05$ en la prueba de igualdad bilateral de proporciones de las columnas. Las casillas sin subíndices no se incluyen en la prueba. Las pruebas asumen varianzas iguales.

Tabla 3. Matriz de componentes principales con una dimensión (pesos factoriales)

\begin{tabular}{|llrrrr|}
\hline & Es capaz de... & PFAQ Original & & PFAQ-7Ch \\
F1 & Manejar su propio dinero & ECV2010 & EEV2012 & ECV2010 & EEV2012 \\
ECV2013
\end{tabular}

Fuente: ECV2010 ( $n=1.954) ; \operatorname{EEV} 2012(n=1.371)$; ECV2013 $(n=2.644)$.

y F10 "recordar compromisos y acontecimientos familiares", por lo que se decidió eliminarlos en la escala PFAQ modificada.

En ECV2010 el ítem F11 "saludar a los amigos"tiene peso factorial más bajo, mientras que en EEV2012 esto ocurre con el ítem F1 "manejar su dinero". Se probaron ambas versiones de la escala eliminando o no estos ítems, encontrándose que cuando se elimina F11 se obtienen mejores propiedades métricas.

Por lo tanto, la propuesta de escala breve (en adelante "PFAQ-7Ch") elimina los ítems F8, F9, F10 y F11. Respecto de validez de constructo, la escala PFAQ-7Ch se comporta de manera unidi- 
mensional, con pesos factoriales altos para todos los ítems en todas las encuestas analizadas (Tabla 3). El coeficiente Alfa de Cronbach es de 0,924 para PFAQ original y de 0,918 para la PFAQ-7Ch. Si se elimina cualquier otro ítem de esta última escala, no aumenta el coeficiente alfa.

En la muestra agregada de ECV2010 y EEV2012, PFAQ-7Ch tiene un promedio de 1,29, con una desviación estándar de 3,4 , en un rango de puntaje entre 0 y 21 , donde $11,4 \%$ es calificado con déficit si el punto de corte se establece en 4 o más puntos. Esto es consistente con 11,6\%con déficit que sería estimado con PFAQ original (Tabla 4).

Para analizar la validez de los instrumentos, se procedió a calcular sensibilidad y especificidad respecto al punto de corte de $6+$ puntos de la escala original (gold standard) propuesta por Quiroga, Albala y Kleesen ${ }^{11}$ para Chile. La sensibilidad es de 0,904 y de 0,877 y la especificidad de 0,989 y de 0,990 para ECV2010 y EEV2012 respectivamente (Tabla 5). Cabe destacar que en PFAQ-7Ch prácticamente ninguna persona calificada "sin déficit" tiene alguna incapacidad en alguna de las actividades medidas (Tabla 5); sin embargo, entre los que son clasificados con déficit, hay mayor variabilidad, donde $27 \%$ es incapaz de realizar las siete actividades y $12 \%$ tiene incapacidad en seis actividades. La actividad en que más se tiene dificultad entre las personas calificadas con déficit es "hacer las compras" (sólo 13\% de los con déficit es capaz de hacerla), mientras que la actividad que más frecuentemente se puede hacer entre los clasificados con déficit es "manejar su propio dinero" (44\%). Lo anterior también es consistente con que las correlaciones ítem-escala son relativamente altas, siendo la menor respecto de "manejar el dinero" $(0,779)$ y la mayor respecto de "calentar agua" $(0,860)$ (Tabla 6$)$.

Respecto de la validez convergente, para analizar la precisión, variabilidad y nivel de discriminación de la escala modificada, se procedió a analizar si ésta es capaz de discriminar por edad y por salud. En los cuatro indicadores analizados (Tabla 7) tanto la escala PFAQ original como PFAQ-7Ch

Tabla 4. Estadísticos descriptivos de las escalas PFAQ original y PFAQ-7Ch

\begin{tabular}{|lcccccc|}
\hline & ECV2010 & $\begin{array}{c}\text { PFAQ Original } \\
\text { EEV2012 }\end{array}$ & ECV2013 & ECV2010 & $\begin{array}{c}\text { PFAQ-7Ch } \\
\text { EEV2012 }\end{array}$ & ECV2013 \\
Media & 2,13 & 2,02 &. & 1,36 & 1,21 & 1,27 \\
Mínimo & 0 & 0 &. & 0 & 0 & 0 \\
Máximo & 33 & 33 &. & 21 & 21 & 21 \\
Desviación típica & 5,04 & 4,47 &. & 3,44 & 2,93 & 3,59 \\
Porcentaje con déficita & $11,80 \%$ & $11,20 \%$ & & $11,90 \%$ & $10,80 \%$ & $11,30 \%$ \\
\hline
\end{tabular}

Fuente: ECV2010 ( $n=1.954)$; EEV2012 $(n=1.371)$; ECV2013 $(n=2.644)$. aPunto de corte escala PFAQ original: $6+$ con déficit; Punto de corte escala PFAQ-7Ch: 4+ con déficit.

Tabla 5. Sensibilidad y especificidad para cada una de las escalas creadas

\begin{tabular}{|lcccccc|}
\hline & \multicolumn{2}{c}{$\begin{array}{c}\text { Sin F7 } \\
\text { (corte en 4) }\end{array}$} & \multicolumn{2}{c}{$\begin{array}{c}\text { Sin F7 y F11 } \\
\text { (corte en 4) }\end{array}$} & \multicolumn{2}{c|}{$\begin{array}{c}\text { Sin F1, F7 y F11 } \\
\text { (corte en 3) }\end{array}$} \\
& ECV2010 & EEV2012 & ECV2010 & EEV2012 & ECV2010 & EEV2012 \\
Eensibilidad & 0,917 & 0,909 & 0,904 & 0,877 & 0,939 & 0,948 \\
Especificidad & 0,987 & 0,985 & 0,989 & 0,990 & 0,963 & 0,969 \\
Valor predictivo positivo & 90,129 & 88,608 & 92,000 & 91,837 & 77,338 & 79,348 \\
Valor predictivo negativo & 98,888 & 98,846 & 98,718 & 98,448 & 99,158 & 99,326 \\
Valor predictivo global & 97,836 & 97,666 & 97,939 & 97,739 & 96,033 & 96,645 \\
Razón verosimilitud positiva & 68,259 & 61,465 & 85,974 & 88,904 & 25,513 & 30,363 \\
\hline Razón verosimilitud negativa & 0,084 & 0,092 & 0,097 & 0,125 & 0,063 & 0,054 \\
\hline
\end{tabular}

Fuente: $\operatorname{ECV} 2010$ ( $n=1.954) ; \operatorname{EEV} 2012(n=1.371) ; \operatorname{ECV} 2013(n=2.644)$. 
Validación test capacidad funcional en adultos mayores - M. S. Herrera et al

discriminan adecuadamente en la dirección esperada: el porcentaje de personas identificadas con déficit funcional es significativamente mayor después de los 75 años, a medida que se tiene peor percepción de salud, si ha sufrido algún problema de salud importante en el último año y si ha tenido limitaciones físicas que le impiden hacer cosas que antes le gustaba hacer.
En relación a la validez predictiva, si bien los datos provienen de estudios transversales no longitudinales, cabe destacar que la escala PFAQ-7Ch también se asocia de manera estadísticamente significativa con otras variables que se supone están afectadas por una disminución de la capacidad funcional (Tabla 8): las personas con menor funcionalidad o con presencia de déficit en la escala

Tabla 6. Cruces entre escala PFAQ-7Ch y cada uno de sus ítems

\begin{tabular}{|c|c|c|c|c|}
\hline Sin déficit & & \multicolumn{2}{|c|}{$\begin{array}{l}\text { PFAQ-7Ch } \\
\text { Sin déficit }\end{array}$} & \multirow{2}{*}{$\begin{array}{c}\text { Correlaciones } \\
\text { ítem-escala } \\
, 779^{* *}\end{array}$} \\
\hline Es capaz de manejar su propio dinero & $\begin{array}{l}\text { Sí es capaz o podría hacerlo } \\
\text { No es capaz }\end{array}$ & $\begin{array}{r}98,5 \% \\
0,1 \%\end{array}$ & $\begin{array}{l}43,9 \% \\
12,2 \%\end{array}$ & \\
\hline $\begin{array}{l}\text { Es capaz de calentar agua para café o té y } \\
\text { apagar la cocina }\end{array}$ & $\begin{array}{l}\text { Sí es capaz o podría hacerlo } \\
\text { No es capaz }\end{array}$ & $\begin{array}{r}99,0 \% \\
0,0 \%\end{array}$ & $\begin{array}{l}35,4 \% \\
20,1 \%\end{array}$ & $860^{\star *}$ \\
\hline $\begin{array}{l}\text { Es capaz de manejar sus propios } \\
\text { medicamentos }\end{array}$ & $\begin{array}{l}\text { Sí es capaz o podría hacerlo } \\
\text { No es capaz }\end{array}$ & $\begin{array}{r}97,7 \% \\
0,1 \%\end{array}$ & $\begin{array}{l}33,1 \% \\
16,8 \%\end{array}$ & $839^{* *}$ \\
\hline $\begin{array}{l}\text { Es capaz de quedarse solo en la casa sin } \\
\text { problemas }\end{array}$ & $\begin{array}{l}\text { Sí es capaz o podría hacerlo } \\
\text { No es capaz }\end{array}$ & $\begin{array}{r}97,4 \% \\
0,1 \%\end{array}$ & $\begin{array}{l}33,7 \% \\
20,2 \%\end{array}$ & $824^{* *}$ \\
\hline $\begin{array}{l}\text { Es capaz de mantenerse al tanto de los } \\
\text { acontecimientos y de }\end{array}$ & $\begin{array}{l}\text { Sí es capaz o podría hacerlo } \\
\text { No es capaz }\end{array}$ & $\begin{array}{r}95,5 \% \\
0,3 \%\end{array}$ & $\begin{array}{l}28,8 \% \\
18,7 \%\end{array}$ &, $787^{* *}$ \\
\hline Es capaz de hacer las compras & $\begin{array}{l}\text { Sí es capaz o podría hacerlo } \\
\text { No es capaz }\end{array}$ & $\begin{array}{r}93,6 \% \\
0,4 \%\end{array}$ & $\begin{array}{l}13,3 \% \\
26,9 \%\end{array}$ & $815^{* *}$ \\
\hline $\begin{array}{l}\text { Es capaz de andar por el vecindario y } \\
\text { encontrar el camino de }\end{array}$ & $\begin{array}{l}\text { Sí es capaz o podría hacerlo } \\
\text { No es capaz }\end{array}$ & $\begin{array}{r}97,7 \% \\
0,0 \%\end{array}$ & $\begin{array}{l}25,5 \% \\
22,3 \%\end{array}$ & $851^{* *}$ \\
\hline Número promedio de actividades en que N & es capaz & 0,29 & 4,7 & \\
\hline Porcentaje NO capaz de realizar las siete act & ividades & $0 \%$ & $27 \%$ & \\
\hline Porcentaje capaz de realizar todas las activi & lades & $80 \%$ & $0 \%$ & \\
\hline
\end{tabular}

Fuente: Base agregada ECV2010, EEV2012 y ECV2013 ( $\mathrm{n}=5.969)$. Nota: Se han excluido las categorías intermedias de cada uno de los ítems, por esto las columnas no suman $100 \%$.

Tabla 7. Validez concurrente ( $\%$ con déficit y promedio escala)

\begin{tabular}{|c|c|c|c|c|c|c|c|c|c|}
\hline & \multicolumn{2}{|c|}{ Edad (1) } & \multicolumn{3}{|c|}{ Percepción de salud (1) } & \multicolumn{2}{|c|}{$\begin{array}{l}\text { En el último } \\
\text { año: Ha sufrido } \\
\text { un problema } \\
\text { de salud } \\
\text { importante (2) }\end{array}$} & \multicolumn{2}{|c|}{$\begin{array}{l}\text { En el último año: } \\
\text { Ha tenido limitaciones } \\
\text { físicas que le impiden } \\
\text { hacer cosas que antes } \\
\text { le gustaba hacer (2) }\end{array}$} \\
\hline & $60-74$ & 75 y más & Buena & Regular & Mala & No & Sí & No & Sí \\
\hline $\begin{array}{l}\text { Escala Pfeffer } \\
\text { Original }\end{array}$ & $\begin{array}{l}5,9 \%_{a} \\
1,10 a\end{array}$ & $\begin{array}{c}20,6 \%{ }_{b} \\
3,67_{b}\end{array}$ & $\begin{array}{l}5,0 \% \\
1.04 a\end{array}$ & $\begin{array}{c}13,3 \%{ }_{b} \\
2.26_{b}\end{array}$ & $\begin{array}{c}26,6 \%_{c} \\
4.78_{c}\end{array}$ & $\begin{array}{l}6,2 \% \\
1.19\end{array}$ & $\begin{array}{c}19,6 \%_{b} \\
3.41_{b}\end{array}$ & $\begin{array}{l}3,7 \% \text { a } \\
.81_{a}\end{array}$ & $\begin{array}{c}19,6 \%{ }_{b} \\
3.36_{b}\end{array}$ \\
\hline $\begin{array}{l}\text { Escala Pfeffer } \\
\text { Modificada }\end{array}$ & $\begin{array}{l}, 63 \mathrm{a} \\
5,6 \%\end{array}$ & $\begin{array}{c}2.30_{b} \\
20,4 \% b\end{array}$ & $\begin{array}{c}.69 \\
6,0 \%\end{array}$ & $\begin{array}{c}1.27 \mathrm{~b} \\
11,7 \% \mathrm{~b}\end{array}$ & $\begin{array}{c}2.84_{c} \\
24,4 \%_{c}\end{array}$ & $\begin{array}{r}.666_{a} \\
5,9 \% a\end{array}$ & $\begin{array}{c}2.13_{b} \\
19,1 \%_{b}\end{array}$ & $\begin{array}{c}.47 \\
4,0 \%\end{array}$ & $\begin{array}{c}2.03_{b} \\
18,4 \% b\end{array}$ \\
\hline
\end{tabular}

Fuente: (1) Base agregada ECV2010, EEV2012 y ECV2013 ( $n=5.969) ;(2)$ EEV2012 ( $n=1.371)$. En la primera fila se expone el $\%$ con déficit y en la segunda el promedio de la escala. Nota: Los valores de la misma fila y subtabla que no comparten el mismo subíndice son significativamente diferentes en $p<, 05$ en la prueba de igualdad bilateral de proporciones de las columnas. Las casillas sin subíndices no se incluyen en la prueba. Las pruebas asumen varianzas iguales. 
Validación test capacidad funcional en adultos mayores - M. S. Herrera et al

Tabla 8. Validez predictiva (\% con déficit y promedio escala)

\begin{tabular}{|c|c|c|c|c|c|c|c|c|c|c|}
\hline & \multicolumn{2}{|c|}{$\begin{array}{l}\text { Percepción de } \\
\text { satisfacción con } \\
\text { la vida (1) }\end{array}$} & \multicolumn{2}{|c|}{$\begin{array}{c}\text { ¿Se siente } \\
\text { frecuentemente } \\
\text { desvalido o que } \\
\text { no vale nada? (1) }\end{array}$} & \multicolumn{3}{|c|}{$\begin{array}{c}\text { ¿Cuán capaz se ha } \\
\text { sentido Ud. para } \\
\text { enfrentar hechos difíciles } \\
\text { que le ha tocado vivir? (2) }\end{array}$} & \multicolumn{3}{|c|}{$\begin{array}{l}\text { En el último año, ¿ha } \\
\text { confiado en que si se es- } \\
\text { fuerza, puede conseguir } \\
\text { lo que desea? (2) }\end{array}$} \\
\hline & $\begin{array}{l}\text { Poco } \\
\text { satisfe- } \\
\text { cho }\end{array}$ & $\begin{array}{l}\text { Satisfe- } \\
\text { cho }\end{array}$ & Sí & No & $\begin{array}{l}\text { Muy } \\
\text { capaz }\end{array}$ & $\begin{array}{l}\text { Poco } \\
\text { capaz }\end{array}$ & $\begin{array}{l}\text { Sin } \\
\text { capaci- } \\
\text { dad }\end{array}$ & $\begin{array}{l}\text { Bas- } \\
\text { tante } \\
\text { seguido }\end{array}$ & $A$ veces & $\begin{array}{c}\text { Casi } \\
\text { nunca }\end{array}$ \\
\hline $\begin{array}{l}\text { Escala Pfeffer } \\
\text { Original }\end{array}$ & $\begin{array}{c}18,0 \% \\
3,15_{a}\end{array}$ & $\begin{array}{l}7,2 \%{ }_{b} \\
1,37_{b}\end{array}$ & $\begin{array}{c}32,6 \% \\
5,63{ }_{a}\end{array}$ & $\begin{array}{l}6,8 \%{ }_{b} \\
1,30_{b}\end{array}$ & $\begin{array}{l}5,3 \%{ }_{a} \\
1,02 a\end{array}$ & $\begin{array}{c}23,6 \% b \\
3,70_{b}\end{array}$ & $\begin{array}{l}54,9 \%_{c} \\
11,29_{c}\end{array}$ & $\begin{array}{c}4,2 \%{ }_{a} \\
, 82_{a}\end{array}$ & $\begin{array}{c}15,3 \%{ }_{b} \\
2,55_{b}\end{array}$ & $\begin{array}{c}38,0 \%{ }_{c} \\
7,11_{c}\end{array}$ \\
\hline $\begin{array}{l}\text { Escala Pfeffer } \\
\text { Modificada }\end{array}$ & $\begin{array}{c}16,6 \%_{a} \\
1,91_{a}\end{array}$ & $\begin{array}{c}8,0 \% \mathrm{~b} \\
, 89_{\mathrm{b}}\end{array}$ & $\begin{array}{c}30,5 \% \\
3,54 a\end{array}$ & $\begin{array}{c}6,9 \% \mathrm{~b} \\
, 76_{\mathrm{b}}\end{array}$ & $\begin{array}{c}4,2 \% \text { a } \\
, 61_{a}\end{array}$ & $\begin{array}{c}15,3 \%_{b} \\
2,31_{b}\end{array}$ & $\begin{array}{c}38,0 \%_{c} \\
7,56_{c}\end{array}$ & $\begin{array}{l}4,3 \%{ }_{a} \\
, 50 a\end{array}$ & $\begin{array}{c}15,7 \% \%_{b} \\
1,63_{b}\end{array}$ & $\begin{array}{c}37,2 \%_{c} \\
4,59_{c}\end{array}$ \\
\hline
\end{tabular}

Fuente: (1) Base agregada ECV2010, EEV2012 y ECV2013 ( $n=5.969) ;(2)$ ECV2010 ( $n=1.954)$. En la primera fila se expone el \% con déficit y en la segunda el promedio de la escala. Fuente: Nota: Los valores de la misma fila y subtabla que no comparten el mismo subíndice son significativamente diferentes en $\mathrm{p}<, 05$ en la prueba de igualdad bilateral de proporciones de las columnas. Las casillas sin subíndices no se incluyen en la prueba. Las pruebas asumen varianzas iguales.

PFAQ-7Ch están menos satisfechos con su vida, se han sentido más frecuentemente "desvalido o que no vale nada", declaran menor "capacidad para enfrentar los hechos difíciles que les ha tocado vivir" y han confiado menos en que "si se esfuerza puede conseguir lo que desea”.

\section{Discusión}

Nuestro estudio confirmó la unidimensionalidad de la escala y demostró que se puede utilizar una escala más breve donde se eliminan cuatro ítems de la versión original validada en Chile por Quiroga, Albala y Klaasen ${ }^{11}$. Se eliminó el ítem "Preparar la comida” por tener sesgo de género, pues en Chile son muchos los hombres -especialmente mayores- que no saben preparar la comida porque nunca lo han hecho, no porque hayan perdido esta capacidad en la vejez. En segundo lugar, se eliminaron tres ítems que se correlacionaban más bajos con la escala: i) "Poner atención, entender, discutir un programa de radio, televisión o un artículo del periódico", el cual seguramente tenía baja correlación porque es una frase muy larga y porque el término "discutir" en Chile puede entenderse no sólo como capacidad de dialogar sobre un tema (acepción original) sino también como "pelear"; además es la única actividad que es más desarrollada por los hombres, por lo que también podría tener cierto sesgo de género. El efecto del posible doble significado de determinadas palabras ha sido encontrado en otras investigaciones, por ejemplo, en la frase "preocupación por la muerte" en la encuesta de la calidad de vida WHOQoL-Old" ${ }^{12}$; ii) "Recordar compromisos y acontecimientos familiares", tiene más baja correlación probablemente porque es una actividad que no depende tanto de la edad, pues hay personas jóvenes que suelen no recordar este tipo de acontecimientos; y iii) "Saludar a sus amigos adecuadamente", es una pregunta que tiene poca variabilidad, pues la mayoría se declara capacitada para realizarla.

La mayoría de las investigaciones sólo han validado la escala para población institucionalizada o con problemas de salud ${ }^{13-15}$. La escala aquí propuesta, en cambio, sirve para discriminar en población adulta mayor general que contesta encuestas, se correlaciona con variables que se suponen miden pérdida de funcionalidad (salud) y con variables con que debería estar asociada (bienestar, autoeficacia) ${ }^{16,17}$.

Cabe igualmente preguntarse si hay diferencias en el porcentaje de personas con déficit funcional cuando se calcula en la muestra de este estudio, realizada en quienes viven en sus viviendas y que sí pueden contestar una encuesta, en comparación con una encuesta más amplia que incluye también a los que no pueden contestar encuestas. Los resultados de este estudio son similares a los estimados a partir de la Encuesta Nacional de Dependencia en Chile del año $2009^{18}$, que es representativa de todos los adultos mayores chilenos (que pueden y que no pueden contestar encuesta): en ésta se estimó en $12 \%$ los adultos mayores con 
Validación test capacidad funcional en adultos mayores - M. S. Herrera et al

déficit funcional y en sólo $2 \%$ la cifra de personas mayores que no pueden contestar por sí mismos una encuesta.

Así como se exploró la asociación de la escala con género para descartar posibles sesgos culturales, se profundizó también en la relación con educación. Todos los ítems se asocian con educación. Pero esto no necesariamente representa un sesgo cultural, porque en Chile varios estudios han mostrado que el estado de salud de las personas no sólo depende de la edad sino también de la educación ${ }^{11,16,17}$. Sin embargo, en relación con lo anterior, hay que considerar también que el hecho de que la escala no presente sesgos culturales de género no quita que pueda presentar otros sesgos que no se han considerado en este artículo, por ejemplo, con etnia o ruralidad (las muestras de estas encuestas no permiten hacer estas distinciones).

Dentro de las limitaciones que presenta la escala propuesta, debe considerarse que si bien logra discriminar a los que tienen déficit (pues se asocia con edad y salud, y predice bienestar y autoeficacia), al obtenerse casi $90 \%$ de adultos mayores sin déficit no está discriminando en grados de capacidad funcional entre los que no tienen déficit, y debido a ello, tampoco identifica a quienes están en riesgo de perder funcionalidad.

En conclusión, se puede decir que la investigación cumple con el objetivo de presentar una propuesta más reducida del instrumento y de más fácil aplicación, válida en contextos de población adulta mayor que puede contestar por sí sola.

\section{Referencias}

1. Naciones Unidas. América Latina y El Caribe: Observatorio Demográfico. Proyecciones de Población. Santiago de Chile: Comisión Económica para América Latina y El Caribe (CEPAL); 2013.

2. CEPAL. Envejecimiento Demográfico. Observatorio Demográfico no 12. Santiago de Chile: Comisión Económica para América Latina y El Caribe (CEPAL); 2011.

3. OECD. Brief Notes OECD Health Statistics-Chile en comparación. http://www.oecd.org/health/healthdata 2013.

4. Mc Dowell I. Measuring health: A guide for rating scales and questionnaires. New York: Oxford University Press; 2006 (third edition).

5. CELADE. Manual sobre Indicadores de Calidad de Vida en la Vejez. Santiago de Chile: Centro Latinoamericano y Caribeño de Demografía (CELADE)-División de Población, CEPAL; 2006.

6. Katz S, Downs TD, Cash HR, Grotz R.Progress in development of the Index of ADL. Gerontologist 1970; 10: 20-30.

7. Mahoney FI, Wood OH, Barthel DW. Rehabilitation of chronically ill patients: the influence of complications on the final goal. South Med J 1958; 51: 605-9.

8. Lawton M, Brody E. Assessment of older people: Self maintaining and instrumental activities of daily living. Gerontologist 1989; 9: 179-83.

9. Pfeffer RI, Kurosaki TT, Harrach CH, Chance JM, Filos $\mathrm{S}$. Measurement of functional activities in older adults in the community. J Gerontology 1982; 37: 323-9.

10. Pfeffer RI, Kurosaki TT, Chance JM, Filos S, Bates D.Use of the mental function index in older adults: reliability, validity, and measurement of change over time. Am J Epidemiol 1984; 120: 922-35.

11. Quiroga P, Albala C, Klaasen G. [Validation of a screening test for age associated cognitive impairment, in Chile]. Rev Med Chile 2004; 132: 467-78.

12. Urzúa A, Navarrete M. Calidad de vida en adultos mayores: análisis factoriales de las versiones abreviadas del WHOQoL-Old en población chilena. Rev Med Chile 2013; 141: 28-33.

13. Hershey LA, Jaffe DF, Greenough PG, Yang SL. Validation of cognitive and functional assessment instruments in vascular dementia. Int J Psychiatry Med 1987; 17: 183-92.

14. Senanarong V, Cummings JL, Fairbanks L, Mega M, Masterman DM, O'Connor SM, Strickland TL. Agitation in Alzheimer's disease is a manifestation of frontal lobe dysfunction. Dement Geriatr Cogn Disord 2004; 17: 14-20.

15. Olazaran J, Mouronte P, Bermejo F. Validez clínica de dos escalas de actividades instrumentales en la enfermedad de Alzheimer. Neurología 2005; 20:395-401.

16. Herrera MS, Barros C, Fernández MB. Predictors of Quality of Life in Old Age: A Multivariate Study in Chile. Journal of Population Ageing 2011;4(3):121-39.

17. Carrasco M, Herrera MS, Fernández MB, Barros C. Impacto del apoyo familiar en la presencia de quejas depresivas en personas mayores chilenas. Rev Esp Geriat Gerontol 2013; 48 (1): 9-14.

18. SENAMA. Encuesta Nacional de Dependencia en las Personas Mayores 2009. Santiago de Chile: Servicio Nacional del Adulto Mayor, Instituto de Nutrición y Tecnología de los Alimentos y Microdatos Universidad de Chile; 2010. 